\title{
Dietary modification of B lymphocytes
}

\author{
B. E. Martínez-Carrillo ${ }^{1,2}$, R. A. Jarillo-Luna ${ }^{2}$, V. Rivera-Aguilar ${ }^{2}$, R. Campos-Rodríguez ${ }^{2}$, \\ R. V. Pardo-Morales ${ }^{1}$, C. Rosales-Gómez and R. Valdés-Ramos ${ }^{1}$ \\ ${ }^{1}$ Center for Research and Graduate Studies of Health Sciences, Faculty of Medicine, Universidad Autónoma del Estado \\ de México and ${ }^{2}$ Instituto Politécnico Nacional, Laboratorio de Inmunología
}

The immune system has evolved as a defence mechanism to maintain good health. In recent years, the effect of diet on various aspects of immune function has become increasingly apparent ${ }^{(1)}$. These effects have also been observed on some areas of the immune system, however, little is known about changes caused by diet on the gut mucosal immunity, which is the first spot of entry for dietary components and particularly with respect to humoral immunity ${ }^{(2)}$. The objective of this study was to evaluate the effect of a high fat (HFD) or high carbohydrate (HCD) diet on B and IgA + lymphocytes in the murine mucosal immune system. Twenty-one day old Balb/c mice were fed a HFD $(23.60 \%$ lard, $40.26 \%$ carbohydrate and protein $23.30 \%)$, a HCD (4.3\% lard, $66.35 \%$ carbohydrate and $18.95 \%$ protein) or a Control diet $(7.01 \%$ lard, $62.94 \%$ carbohydrate and protein $20 \%)$ during 9 weeks $(n=8)$, after which small intestine and Peyer's patches were obtained, for purification and phenotyping of B-lymphocytes and IgA + cells by flow cytometry. Mean CD19+ cells in lamina propria for controls was $15.71 \pm 2.87 \%$, decreasing in both HFD and HCD $(9.09 \pm 2.52 \%, 6.94 \pm 1.50 \%$ respectively); statistically significant differences were observed by ANOVA $(F=29.61, P<0.0001)$. Peyer's patches showed highest values of CD19+ cells in the controls $(69.23 \pm 2.41 \%)$, followed by HCD and HFD $(43.30 \pm 6.54 \%$ and $50.69 \pm 6.06 \%$, respectively), differences were statistically significant $(F=71.96$ and $P<0.0001)$ (Fig. 1). IgA + cells in lamina propria were highest in the HCD group $(45.57 \pm 5.59 \%)$, followed by HFD $(31.42 \pm 5.36 \%)$ and Controls $(22.66 \pm 0.92 \%)(F=48.97$ and $P<0.0001)$. Peyer's patches IgA + cells were highest in the HFD group $(59.05 \pm 7.66 \%)$, followed by HCD $(38.72 \pm 5.24)$ and controls $(23.58 \% \pm 2.14)$ (ANOVA $F=50.20, P<0.0001)($ Fig. 2$)$. Both diets decreased CD19+ cells in lamina propria and Peyer's patches, whereas $\operatorname{IgA}+$ cells were higher. It is possible that these dietary modifications are either decreasing the presence of B lymphocytes or accelerating their transformation in IgA producing plasma cells, acting as an antigenic stimulus at the mucosal levels. This project was financed by Universidad Autónoma del Estado de México.

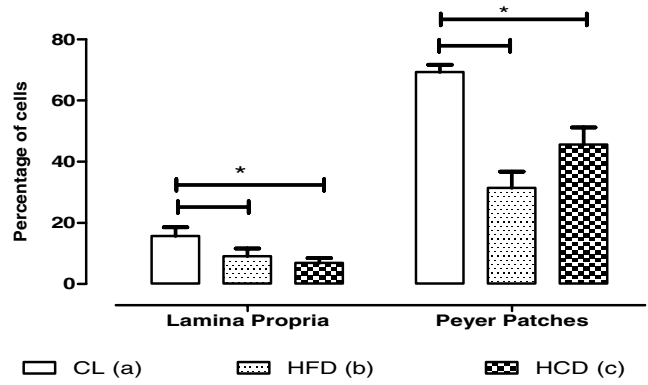

Fig. 1. Percentage of B-lymphocytes $(\mathrm{CD} 19+)$ in lamina propria and Peyer's patches of $\mathrm{Balb} / \mathrm{c}$ mice fed with three diets. The values represent the means $\pm \mathrm{SD}$ of three determinations, each group with $n=8$, ANOVA, $P<0.0001$. *Post hoc Tukey test comparing the three groups $(\mathrm{a}, \mathrm{b}$ and, $\mathrm{c})$ showed differences between groups with an IC of $95 \%, P<0.0001$; (a) CL (control), (b) HFD (high fat diet) and (c) HCD (high carbohydrate diet)

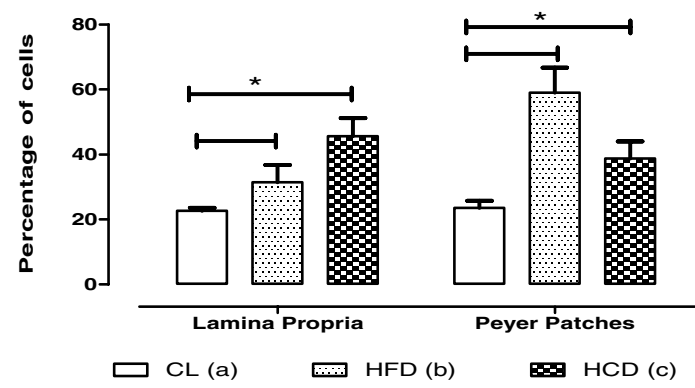

Fig. 2. Percentage of IgA + cells in lamina propria and Peyer's patches in Balb/c mice fed with three diets. The values represent the means \pm SD of three determinations, each group with $n=8$, ANOVA, $P<0.0001$ * Post hoc Tukey test comparing the three groups (a, b and c) showed differences between groups, with an IC of $95 \%$, $P<0.0001$; (a) CL (control), (b) HFD (high fat diet) and (c) HCD (high carbohydrate diet), IgA (immunoglobulin A) 